\title{
Charles Darwin y el "desencantamiento" weberiano
}

\author{
Charles Darwin and Weberian "Disenchantment"*
}

\author{
BÁRBARA JIMÉNEZ PAZOS**
}

\begin{abstract}
Resumen: Este artículo pretende poner de manifiesto la interrelación permanente entre dos metáforas darwinianas como "la orilla enmarañada", presente en On the Origin of Species, y la relativa al "daltonismo", mencionada en Autobiography, para demostrar la dudosa consistencia de interpretaciones que detectan un "encanto" secular latente en la Teoría de la Evolución y descartan un "desencantamiento", en términos weberianos (Entzauberung).

Palabras clave: Darwin, Entzauberung, naturaleza, percepción de la naturaleza, estética, sublime.
\end{abstract}

\begin{abstract}
The aim of this article is to reveal the permanent interrelation between two Darwinian metaphors like the "entangled bank" in On the Origin of Species, and the one concerning the "color-blindness" mentioned in Autobiography, in order to demonstrate the doubtful consistency of interpretations which detect a latent secular "enchantment" in the Theory of Evolution, and reject a "disenchantment", in weberian terms (Entzauberung).
\end{abstract}

Keywords: Darwin, Entzauberung, nature, perception of nature, aesthetics, sublime.

\section{La orilla enmarañada, metáfora del desencantamiento positivo ${ }^{1}$}

Wie die Rococo-Gartenkunst entstand, aus dem Gefühl ,die Natur ist hässlich, wild, langweilig, auf! wir wollen sie verschönern (embellir la nature)!“” - so entsteht aus dem Gefühl ,die Wissenschaft ist hässlich, trocken, trostlos, schwierig, langwierig, - auf! Lasst uns sie verschönern!“ immer wieder Etwas, das sich die Philosophie nennt (Fr. Nietzsche, Morgenröthe, §427)

Fecha de recepción:18/09/2015. Fecha de aceptación: 15/10/2015.

* Este artículo se ha realizado gracias a una beca predoctoral concedida por la UPV/EHU (PIF UPV 012/2011).

** Doctora en Filosofía e investigadora postdoctoral en la Universidad del País Vasco, Departamento de Filosofía (San Sebastián). E-mail: barbara.jimenez@ehu.eus. Líneas de investigación: Darwinismo e Imagen del Mundo, Romanticismo Inglés, Percepción y Descripción del Paisaje Natural, Historia de las Ideas, Historia de la Ciencia. Publicaciones recientes: "Metaphysics in the Work of Charles Darwin", in Metaphysics or Modernity, edited by S. Baumgartner, T. Heisenberg and S. Krebs, Bamberg, University of Bamberg Press, 2013; "Percepción, Descripción y Explicación en la Obra de Alexander von Humboldt”, Ideas y Valores 165 (en prensa, diciembre de 2017).

1 Abreviaturas: Teoría de la Evolución: TE. 
El último párrafo de On the Origin of Species (1859) resulta clave para un estudio centrado en la percepción darwiniana de la belleza natural:

It is interesting to contemplate an entangled bank, clothed with many plants of many kinds, with birds singing on the bushes, with various insects flitting about, and with worms crawling through the damp earth, and to reflect that these elaborately constructed forms, so different from each other, and dependent on each other in so complex a manner, have all been produced by laws acting around us [...] There is grandeur in this view of life, with its several powers, having been originally breathed into a few forms or into one; and that, whilst this planet has gone cycling on according to the fixed law of gravity, from so simple a beginning endless forms most beautiful and most wonderful have been, and are being, evolved ${ }^{2}$

Las múltiples interpretaciones expertas con respecto al sentido de las palabras del naturalista han oscilado, lógicamente, entre la defensa de una visión mecanizada del mundo fundada en las leyes que componen la Teoría de la Evolución (TE) darwiniana, y una posición que pretende destacar la maravillosa "grandeza" y "belleza" que alberga la naturaleza con orillas enmarañadas, "cubiertas con plantas de varios tipos, pájaros cantando en los arbustos, diferentes insectos revoloteando, y con gusanos arrastrándose por la arena húmeda". Sin embargo, las leyes que fundan la TE, traídas hasta las líneas finales del Origen y enumeradas una a una ("Crecimiento con Reproducción", "Herencia", "Variación", "Proporción del Aumento", "Lucha por la Vida", "Selección Natural", "Divergencia de Caracteres", "Extinción"3) parecen susceptibles de interpretaciones dicotómicas como las que defienden un encanto implícito en la TE, por un lado, y por otro, las que destacan la preponderancia del carácter científico de forma exclusiva, derivable en un posible efecto desencantador. El "desencantamiento" (Entzauberung) que en Wissenschaft als Beruf (1919) Max Weber atribuye a la explicación racionalizada del mundo que proporcionan las ciencias naturales, radicaría en la capacidad de acceso al conocimiento racional y sometido al cálculo de forma voluntaria y en cualquier momento, indudable desencadenante del desvanecimiento de la creencia en explicaciones mágicas sobre el funcionamiento del mundo:

La creciente racionalización e intelectualización no significa, por tanto, un mayor conocimiento general de las condiciones de vida bajo las que se vive [...] significa el conocimiento o la fe de que, si se quisiera, se podrían conocer en todo momento esas condiciones; significa, por tanto, el conocimiento o la fe de que, por principio, no existen poderes ocultos imprevisibles que estén interviniendo sino que, en principio, se pueden dominar más bien todas las cosas mediante el cálculo. Esto significa, sin embargo, la desmagificación del mundo ${ }^{4}$.

2 Darwin, Ch., On the Origin of Species by Means of Natural Selection, or the Preservation of Favoured Races in the Struggle for Life, London, John Murray, 1859, pp. 489-490. Para referirme a la obra On the Origin of Species utilizaré la abreviatura Origen.

3 Ídem.

4 Weber, M., La Ciencia como Profesión, traducción y edición de Joaquín Abellán, Madrid, Espasa Calpe, 1992, p. 67. Cursivas en el original. Utilizaré el término "desencantamiento" (y no el de "desmagificación", propuesto 
George Levine adopta una posición media entre una interpretación estricta del racionalismo científico darwiniano y su lado más poético. Asimismo, caracteriza la Selección Natural como "re-encantadora", manifestante de leyes diferentes a las divinas que permiten una nueva visión de la sublimidad en la naturaleza, la del movimiento incesante de seres vivos y conexión de fuerzas invisibles aunque latentes y ejecutoras sobre éstos. Levine deshace el desencanto que la teoría de Darwin pudiera suscitar a sus conocedores y propone nuevas formas de encanto secular obtenido a partir de la conciencia "de un mundo abrumadoramente complicado, 'enmarañado', y maravilloso más allá de la imaginación humana"`. La posición de Levine es aquélla que recupera el encanto que supuestamente arrebataría una fijación exclusiva sobre el carácter mecánico de la TE.

Uno de los ejemplos más manifiestos de interpretación "desencantada" de la teoría de Darwin es la de Daniel C. Dennett. Este autor presenta "la peligrosa idea de Darwin"6 como un "ácido universal" que corroe el entramado de ideas tradicionales y ofrece una nueva visión revolucionaria del mundo basada en la "selección natural" considerada como un "algoritmo" mecánico que prescinde de una racionalidad intrínseca y controladora de su propio mecanismo. Levine, como contraste, defendería la importancia de la apreciación romántica de la visión del mundo de Darwin: una nueva experiencia del mundo explicada en términos evolucionistas, "más fascinante, más interesante, más bella"7.

Ciertamente, las interpretaciones que se centran en la racionalidad científica no se detienen sobre la belleza o encanto de la teoría evolutiva darwiniana, y no se muestran receptivas frente a un vocabulario descriptivo que incluya la reverencia o el asombro frente a las fuerzas que funcionan en la naturaleza como características o criterios a tener en cuenta a la hora de observarla y analizarla. Sin embargo, estos aspectos no deben comprenderse como restrictivos y excluyentes. Se tomaría por defecto el indiscutible interés de las teorías de Darwin frente a otro tipo de explicaciones alternativas con base en lo sobrenatural. El carácter enriquecedor de la argumentación científica y la visión del mundo que ésta permite alcanzar, ofrecerían una nueva experiencia cognitiva, admirable en sí misma y cuyo interés habría sido pensado en considerable profundidad.

Levine concede especial importancia a los últimos apuntes del Origen, tomados como referencia clave para vislumbrar una cierta permanencia de lo sublime en las palabras de Darwin sobre la grandeza que percibe en la visión del mundo que propone ${ }^{8}$, y empleados como referencia para disipar todo tipo de interpretación científica árida de las leyes enumeradas $^{9}$ y excluyente de un encanto que el propio Darwin desearía transmitir. Sin duda, Levine no desea emitir un mensaje sobre una interpretación encantada de la las leyes evolutivas en

por algunas traducciones), ya que es el que generalmente se atribuye a la eliminación de "poderes ocultos imprevisibles" descrita por Weber, así como a su efecto de desengaño ilustrado en el ser humano. El desencanto surgiría por la asimilación de que la ciencia moderna no estaría orientada ni habilitada para conferir "sentido" al mundo. Ibíd., pp. 69-74.

5 Levine, G., Darwin Loves You: Natural Selection and the Re-enchantment of the World, Princeton (N.Y.), Princeton University Press, 2008, p. 168.

6 Dennett, D. C., Darwin's Dangerous Idea: Evolution and the Meanings of Life, New York, Simon \& Schuster, 1995.

7 Levine, Darwin Loves You, op. cit., p. 248.

8 "There is grandeur in this view of life". Darwin, Origen, p. 490.

9 “These laws...” Ibíd., 489. 
un sentido estricto, sino que destaca la fascinación que la propia ciencia puede producir sin apelar a explicaciones sobrenaturales. La percepción estética de la naturaleza se vería incluso incrementada, no disminuida, gracias al enfoque científico, promotor de nuevas, profundas y más nítidas aproximaciones al mundo.

Tales ideas ya habían sido avanzadas por Richard Dawkins en su crítica al poeta romántico John Keats y su descontento con las ideas desplegadas en la Óptica (1704) de Newton. Según Keats, la reducción explicativa del arcoíris en términos de longitudes de onda habría despojado de toda poesía al fenómeno lumínico y destruido todo misterio que se pudiese hallar en su contemplación. Dawkins arremete en contra de esta particular comprensión de la Óptica newtoniana, extrapolándola hasta la más general comprensión de la actividad científica hoy en día, y alega a favor de la "purga del empalagoso falso propósito" y el "descrédito del sentimentalismo cósmico"10. En términos de Dawkins, "la ciencia es, o debería ser, la inspiración para la buena poesía" 11 , ofrecería un abanico infinito de posibilidades inspirativas, a cada cual más espectacular y con origen en un misterio ya resuelto, desprendido de toda teleología, sensiblería y oscuridad epistemológica, e inundado de la necesaria claridad explicativa científica que permite desplegar en unidades lo que en un inicio se presentaba caótico y desordenado. Así, Dawkins apuesta por "un universo ordenado, indiferente a las preocupaciones humanas [...] un lugar más bello y maravilloso que un universo embaucado con magia caprichosa y ad hoc"12. La óptica científica, al igual que la newtoniana, conformaría un potenciador idóneo de la percepción estética del mundo, ampliando las sensaciones, aumentando el conocimiento, ofreciendo una belleza más profunda. En último término, mostrando el mundo tal y como esencialmente es, y no tal y como se percibe.

Retrocediendo un paso más en la escala temporal, y pasando por alto múltiples alusiones que cabría tener en cuenta, cabe destacar las palabras de Alexander von Humboldt en su introducción a la obra $\operatorname{Kosmos}^{13}$. Humboldt, basándose en su propia experiencia como naturalista, refuta la extendida idea que sostiene una pérdida de encanto como consecuencia del aumento de conocimiento, propugna la conciliación entre una aproximación científica rigurosa al mundo y el posterior relato que dé cuenta de las impresiones humanas frente a la magnificencia de la naturaleza. El goce obtenido a partir de la observación de la naturaleza podría, según Humboldt, dividirse, por un lado, en un deleite intenso aunque primario frente a la percepción de la existencia de un Todo animado y armónico compuesto de unas leyes físicas intrínsecas cuyo funcionamiento permanecería desconocido para el observador; por otro lado, se encontraría el goce generado a partir del conocimiento exacto y reglado de las características del paisaje, así como de las leyes naturales que operan incesantemente. Considerando ambos tipos de disfrute estético y epistemológico como partes de una progresión necesaria, Humboldt apuesta por el solapamiento de partes en donde deleite estético y conocimiento científico interactúan para generar un tercer tipo de goce, con intensidad racional y estética aumentada.

10 Dawkins, R., Unweaving the Rainbow: Science, Delusion and the Appetite for Wonder, Boston, Houghton Mifflin Company, 1998, p. ix.

11 Ibíd., p. x.

12 Ibíd., p. xi.

13 von Humboldt, A., Kosmos: Entwurf einer physischen Weltbeschreibung, Vol. I (publicado en cinco volúmenes), Stuttgart und Tübingen, Cotta, 1845-1862, pp. 3-48. 
El deleite científico, ya sea promovido por la persistencia romántica según Levine o la potencia explicativa según Dawkins, descansaría en el segundo tipo de goce descrito por Humboldt. La yuxtaposición del conocimiento científico y el goce cognitivo, derivable en una percepción estética potenciada, compone la base sobre la que Darwin apoyaría el peso de la enumeración de sus leyes al final del Origen. La orilla enmarañada a la que Darwin se refiere, constituiría una metáfora de una particular recomposición de elementos que daría cuenta de la existencia del Todo armónico humboldtiano, que ofrecería una explicación racional de las partes y que se detendría para remarcar la grandeza de la visión de la vida que se propone en la obra. El re-encanto que Levine plantea supondría el rechazo de un infundado desencanto por parte de interpretaciones marcadamente cientificistas, reacias a una apreciación más romántica del texto de Darwin. De forma análoga, propugnaría el descrédito de un supuesto desencanto darwiniano destructor de todo sentido y valor en la vida y apoyaría, por el contrario, el encanto secular inherente a la actividad científica. Independientemente del enfoque a partir del cual se estudie la obra de Darwin (el enfoque estrictamente científico o el enfoque filo-romántico), se habría de suponer la existencia de un encanto científico perpetuo y latente en el relato de la TE, no comprendido en un sentido mágico estricto.

Las palabras de Levine se podrían reformular en un sentido inverso, quizás más acorde con el entramado de ideas de Darwin. La TE darwiniana sí ofrece una visión del mundo desencantada, en un sentido estricto del término. El conocimiento de las leyes evolutivas permitiría la observación del mundo a través de un filtro cristalino, con una óptica aumentada, que desmitificaría lo observado y le añadiría valor intelectual y estético, al mismo tiempo. Este desencantamiento habría de comprenderse como una característica favorable para un proceso de reinterpretación de todo lo observado, lejano ya de toda presuposición que implique el uso de imágenes espirituales, mágicas, oscuras desde el punto de vista epistémico, encantadas, en definitiva.

Suponer, a partir de aquí, la existencia de un propósito específico implícito en las palabras que cierran el Origen, implica un cierto riesgo hermenéutico. Sin embargo, las sospechas tienden, obviamente, a relacionar las alusiones a la belleza y el carácter maravilloso de las "infinitas formas" surgidas a partir de un "simple inicio" 14 con el sentimiento de lo sublime en la percepción estética de la naturaleza, en el caso de Darwin, generado a partir de la práctica científica y el hecho de asumir sus resultados. La negatividad que generalmente acompaña al término "desencantamiento" provendría de una asimilación equívoca de las implicaciones que supone el planteamiento de una Teoría como la de Darwin, transgresora por definición, quebrantadora de viejas concepciones del mundo, así como de aceptación de la sospecha de la invasión de un nuevo materialismo adverso a toda metafísica sobrenatural. Según Richard Holmes, “el proceso de evolución por ‘selección natural' reemplazó cualquier necesidad de 'diseño inteligente' en la naturaleza. Darwin ciertamente había escrito un nuevo Libro del Génesis"15. Comprensiblemente, la recepción de tales ideas, dentro de un marco general, no reparaba en apreciaciones de carácter estético a partir de un análisis semiótico del

14 "from so simple a beginning endless forms most beautiful and most wonderful have been, and are being evolved". Darwin, Origen, p. 490.

15 Holmes, R., The Age of Wonder: How the Romantic Generation Discovered the Beauty and Terror of Science, London, Harper Press, 2009, p. 451. 
léxico darwiniano. No obstante, la presunción de la permanencia de un poderoso sentimiento estético proyectado sobre la visión del mundo propuesta por Darwin, habría de permanecer igualmente dudosa.

Existe una evolución de ideas y estilos narrativos fácilmente visible mediante un paralelo avance progresivo y cronológico en la obra de Darwin. La obra Journal of Researches $(1839)^{16}$ representaría un inicial planteamiento, con una narrativa más próxima a la humboldtiana, de lo que culminaría de forma magistralmente desarrollada en el Origen como la TE, planteada con un lenguaje marcadamente científico y no opuesto, aunque sí acentuadamente distante con respecto al del Diario. Simétricamente, las alusiones, características del Diario, a la impresión causada en el observador por ciertas escenas destacadas de la naturaleza y los sentimientos generados al respecto, disminuyen notoriamente en el paso de una obra a la otra. Si las últimas palabras del Origen realmente indican la persistencia y estabilidad de un cierto sentimiento romántico de lo sublime generado, no obstante, a partir de un análisis minucioso del entorno natural, no deberían interpretarse, a mi juicio, como re-encantadoras sino como positivamente desencantadoras y sugerentes de una nueva forma de percepción de la belleza en la naturaleza. El carácter contraintuitivo de la persistencia de un reducto romántico en una progresión visiblemente cientificista, habría de disiparse forzosamente en el caso de que tal vestigio fuese fácilmente proyectable en obras posteriores al Origen y que permitiesen vislumbrar la conformidad del autor con la postura anímica sugerida mediante la metáfora de "la orilla enmarañada".

La comprensión positivamente desencantada que propongo para las últimas líneas del Origen no implica, sin embargo, continuidad prolongada a lo largo del resto de obras de Darwin, sino la percepción de una maniobra ideológica progresivamente opuesta. Las confesiones hechas en Autobiography ${ }^{17}$ indican el deterioro de la forma vívida de percepción estética de la naturaleza, íntimamente ligada a las palabras del Diario e inmortalizada en el Origen mediante ideas evolutivas maduradas. El texto autobiográfico de Darwin revela una angustia frente a un tipo específico de pérdida que él identifica de forma intuitiva como pérdida perceptiva y cuyas implicaciones se situarían cercanas a una comprensión del desencantamiento como proceso negativo, generador de un tipo particular de "daltonismo", que dificultaría la usual forma de aproximación estética al paisaje natural.

\section{El "daltonismo", metáfora del desencantamiento negativo}

Algunas afirmaciones de Darwin en la Autobiografía con respecto a su cambio en la forma de percibir las "escenas" de la naturaleza son especialmente significativas:

I well remember my conviction that there is more in man than the mere breath of his body. But now the grandest scenes would not cause any such convictions and feelings

16 Para referirme a la obra Journal of Researches utilizaré el término Diario como forma abreviada de la traducción Diario del Viaje de un Naturalista alrededor del Mundo. No debe confundirse, sin embargo, con las publicaciones de los diversos diarios que Darwin completó en su viaje a bordo del Beagle.

17 La Autobiografía de Darwin se publica por primera vez como parte de la obra editada por su hijo: The Life and Letters of Charles Darwin, Including an Autobiographical Chapter, edited by Francis Darwin, London, John Murray, 1887. 
to rise in my mind. It may be truly said that I am like a man who has become colourblind, and the universal belief by men of the existence of redness makes my present loss of perception of not the least value as evidence ${ }^{18}$

El recuerdo de Darwin pertenece a la convicción relatada en el Diario, surgida a partir de la observación de escenas elementalmente opuestas, aunque ambas potencialmente generadoras del sentimiento de lo sublime en el observador. La riqueza de formas y elementos que ofrece un paisaje como el de la selva brasileña y el predominio de la "Muerte y Deterioro" de las vastas soledades de Tierra del Fuego, constituyen una evidencia suficiente para afirmar convincentemente la imposibilidad de permanecer impasible frente a tales escenas "y no sentir que hay algo más en el ser humano que el mero aliento de su cuerpo"19. Para asombro de Darwin, lo que años atrás se presentaba como un mecanismo mental automático de observación, percepción y posterior asimilación de lo sublime en la naturaleza, con el paso del tiempo se degrada en lo que el propio autor diagnostica como "daltonismo" perceptivo, una ceguera sentimental cuyos síntomas requieren de un análisis temporal retrospectivo que se retrotrae precisamente a la lectura poética.

El cambio mental del que Darwin se percata $^{20}$ se manifiesta, en primer lugar, en una insólita percepción de la poesía como algo aburrido e incluso nauseabundo, en el caso de Shakespeare ${ }^{21}$. La alteración mental destacada parece encontrar una mención definitiva como "pérdida del gusto", hallada esta vez en la pintura y la música ${ }^{22}$. El género poético, la apreciación pictórica y el gusto musical forman un conjunto que ejerce como preludio de la mención acerca de la percepción paisajística, sobre la cual, Darwin afirma mantener cierto gusto por refinados escenarios naturales. Sin embargo, a su vez, asevera haber perdido la sensación de "deleite exquisito" que tales escenas le solían provocar ${ }^{23}$. La enumeración de ejemplos posee un propósito delimitador centrado sobre la "pérdida de los gustos estéticos más elevados/finos" que, en contraposición con el interés mantenido por lecturas centradas en historia, relatos biográficos, literatura de viajes y ensayos de todo tipo, se calificaría de "curiosa" y "lamentable"24. La curiosidad y angustia mencionadas y, a su vez, proyectadas sobre las palabras de Darwin se deben unir al contenido entre paréntesis ${ }^{25}$, mencionado sutilmente pero cuya presencia no debe pasar desapercibida y exige de reflexión.

Resulta comprensible que se dé en Darwin un deleite por ciertos tipos de narrativa (más ligera, tal vez), no por su contenido científico exclusivamente, sino por constituir un buen

18 Barlow, N. (ed.), The Autobiography of Charles Darwin., 1809-1882, New York, W.W. Norton \& Company, 2005 , p. 76.

19 Darwin, Ch., Journal of Researches into the Natural History and Geology of the Various Countries Visited by H.M.S. Beagle round the World, under the Command of Capt. Fitz Roy, R.N., London, Henry Colburn, 1839, p. 605.

20 "my mind has changed during the last twenty or thirty years". Barlow (ed.), Autobiography, op. cit., p. 112.

21 "I have tried lately to read Shakespeare, and found it so intolerably dull that it nauseated me". Ibíd., p. 113.

22 "I have also lost my taste for pictures or music". Ídem.

23 "I retain some taste for fine scenery, but it does not cause me the exquisite delight which formerly did". Ídem.

24 "This curious and lamentable loss of the higher aesthetic tastes is all the odder, as books on history, biographies, and travels (independently of any scientific facts which they may contain), and essays on all sorts of subjects interest me as much as they did"'. Ídem.

25 "independently of any scientific facts which they may contain". Ídem. 
método de entretenimiento. No obstante, la permanencia del gusto por un tipo específico de narrativa que no es el conjunto de las artes más finas como la poesía, la música, la pintura o el paisajismo, permite distinguir un sentimiento de aturdimiento y deseos de hallar agudeza explicativa sobre lo que le está ocurriendo. A partir de un desconocimiento manifiesto por parte de Darwin, se encuentra en la obra una búsqueda de discernimiento tentativa en la alusión a la posibilidad de hallar un fondo científico en las lecturas sobre las que aún soporta centrarse, un contenido de interés invariable para el autor a lo largo de los años y que conduce hacia la detección de una "atrofia" cerebral parcial, así como a relacionarla con la práctica científica tomada como método exclusivo.

La transformación mental a la que Darwin se refería al inicio de su análisis fáctico recapitulador, consistiría en una supuesta mecanización de la mente, convertida ésta, según sus propias y duras palabras, en "una especie de máquina moledora que extrae leyes generales a partir de una gran recopilación de evidencias" 26 . No obstante, la "parálisis cerebral" vinculada a los gustos más altos, producida aparentemente por una sobre-dedicación al estudio científico, se mantiene como indicio incomprensible ${ }^{27}$, inductor de un desconcierto tan pronunciado que lleva a Darwin a elucubrar sobre posibles métodos que hubiesen evitado el estado de declive perceptivo y emocional que se describe, tales como un acercamiento más regular a la música o la poesía ${ }^{28}$.

Una de las hipótesis más desarrolladas para constituir una estable base explicativa al desorden perceptivo de Darwin, adopta, precisamente, la convicción de que la sobre-concentración en el estudio científico conformaría una de las causas principales de tal desequilibrio. Sin embargo, lo que la aceptación de esta interpretación específica no contempla es el lógico desconcierto del autor frente a las implicaciones explicativas derivadas que tal admisión conllevaría. Aceptando la sobre-dedicación científica como causa de la "atrofia cerebral" de Darwin, habría de argumentarse, en consecuencia, el motivo por el que dicha práctica científica resulta exclusivamente nociva para los gustos más elevados y no para la apreciación y deleite con temas históricos, biográficos o literarios. Esta particular lectura, cegada tal vez por el influjo de la idea weberiana sobre el desencantamiento, resulta resolutivamente tan ineficaz como los vagos intentos del propio Darwin por considerar el seguimiento reglado de hábitos musicales y de lectura poética como base precavida y temporalmente adelantada para eludir todo tipo de atrofias mentales por desuso. El vocabulario del naturalista, no obstante, transmite una potente connotación dubitativa ${ }^{29}$, que deja de ser notoria en la alusión al único conocimiento que parece resultar seguro, a saber, que "la pérdida de estos gustos [los más elevados] es una pérdida de felicidad, y posiblemente sea perjudicial para el intelecto,

26 "My mind seems to have become a kind of machine for grinding general laws out of large collections of facts". Ídem.

27 "why this should have caused the atrophy of that part of the brain alone, on which the higher tastes depend, I cannot conceive". Ídem.

28 "if I had to live my life again, I would have made a rule to read some poetry and listen to some music at least once every week; for perhaps the parts of my brain now atrophied would thus have been kept active through use". Ídem.

29 "why [...] I cannot conceive"; "perhaps". Véase nota 29 y nota 30. 
y probablemente más para el carácter moral, por la debilitación de la parte emocional de nuestra naturaleza" 30 .

La segunda hipótesis que más atención experta ha recibido por ser, quizás, la que mejor da cuenta, precisamente, del origen de la destacada "debilitación de la parte emocional", es la que se centra en el estudio de la modificación de las creencias religiosas del autor. Darwin rememora en su Autobiografía su absoluta convicción de la existencia de Dios y de la inmortalidad del alma citando un pasaje del Diario en donde se destaca el surgimiento de unos sentimientos de "asombro, admiración y devoción" en la selva brasileña de una vehemencia tan pronunciada, que se evadirían de toda descripción ajustada ${ }^{31}$. La incapacidad expresiva, romántica en esencia, frente a la grandeza sublime de la naturaleza, estaría íntimamente ligada al sentimiento de la existencia de "algo más en el ser humano que el mero aliento de su cuerpo". Sin embargo, el "daltonismo" del que Darwin afirma padecer, habría bloqueado el proceso de percepción estética de la naturaleza y posterior brote de sentimientos religiosos.

Las experiencias relatadas en el Diario estarían sustentadas por el cásico vínculo entre el sentimiento de lo sublime y el sentimiento religioso ${ }^{32}$, especialmente encumbrado en las descripciones de la naturaleza del período romántico, un apoyo que habría de desmoronarse con el paso de los años y la progresiva evolución del pensamiento de Darwin. En consecuencia, podría considerarse válida la suposición que contempla el término de un proceso "romántico" de percepción y descripción de la naturaleza dado el análogo fin de la creencia en Dios. Si lo sobrenatural resulta ser el único recurso que puede dar cuenta de lo experimentado frente a ciertas escenas de la naturaleza capaces de generar un sentimiento de sublimidad, y Darwin alude a una irremediable supuesta pérdida de fe, habría de considerarse definitivamente insuficiente una hipotética y deseada constancia, trasladada, en este caso, a la práctica de la observación paisajística, tal y como se sugería para la música y la poesía. No existiría respuesta lógicamente satisfactoria que argumentase cómo un hábito constante en la contemplación estética de la naturaleza habría de deshacer el sentimiento de irreligiosidad manifestado en Darwin.

Deduzco, por consiguiente, la existencia de dos tipos de "pérdidas" perceptivas no contrapuestas, sino solapadas, que permiten vislumbrar fácilmente la presencia de una transición perceptiva y sentimental en Darwin. En primer lugar, se detectaría la referente a la "atrofia cerebral", erróneamente atribuida a una sobre-dedicación a la actividad científica. Por otro lado, se destacaría la producida por un desencantamiento negativo con respecto a la percepción estética paisajística, es decir, un "daltonismo" derivado de la pérdida de creencias religiosas. Matizando este último eslabón del conjunto, cabría precisarlo como modificación de la percepción y habría de desplazarse su comprensión como pérdida. La alteración mental

30 "The loss of these tastes is a loss of happiness, and may possibly be injurious to the intellect, and more probably to the moral character, by enfeebling the emotional part of our nature". Barlow (ed.), Autobiography, op. cit., p. 113.

31 "In my Journal I wrote that whilst standing in the midst of the grandeur of a Brazilian forest, "it is not possible to give an adequate idea of the higher feelings of wonder, admiration, and devotion, which fill and elevate the mind"". Ibíd., p. 76.

32 "The state of mind which grand scenes formerly excited in me, and which was intimately connected with a belief in God, did not essentially differ from that which is often called the sense of sublimity". Ídem. 
de Darwin manifestada en una análoga alteración de la percepción del paisaje natural no es interpretada en la Autobiografía como algo positivo o beneficioso, sino todo lo contrario, a saber, como una variación "perjudicial para el intelecto, y probablemente más para el carácter moral". El desencantamiento que Darwin experimenta, el cese de la creencia en lo religioso, aparentemente acerca al naturalista a una visión más próxima al desencantamiento de connotación negativa sugerido por Max Weber: "atrofia" y "daltonismo" no son términos compatibles con una completa, intensa y positivamente desencantada percepción estética del paisaje, o de forma más específica, con la grandiosa visión del mundo propuesta por Darwin, tal y como se evoca en las líneas conclusivas del Origen.

John A. Campbell hace una lectura de las líneas de la Autobiografía introductoras de la confesión de un declive perceptivo y afectivo basada en una interpretación sostenida en la dicotomía arte/naturaleza que acepta un intenso debilitamiento del interés artístico, pero no del interés por la naturaleza. La nueva visión del mundo basada en la TE habría llevado al naturalista a encontrar un nuevo tipo de grandeza en el análisis minucioso y diferenciado de los elementos de la naturaleza y sería fácilmente observable en el lenguaje empleado en las descripciones de Darwin, revelador de "una relación con sus objetos de estudio que es personal y afectiva" 33 , humildemente respetuosa con todos los elementos componentes de la naturaleza. La posición de Campbell podría equipararse a la de G. Levine y su insistencia por afirmar que "lo maravilloso para Darwin permanece en el mundo natural y en su experiencia de ello"34. Si han de considerarse seriamente las propuestas de ambos autores, habría que ofrecer, por un lado, respuesta al motivo por el que una alusión, por mínima y cautelosa que fuese, al surgimiento de sentimientos de admiración y belleza, modificados en base a una nueva concepción del mundo, permanece ausente en la Autobiografía. Por otro lado, partiendo del supuesto de que Darwin se percatase de la creciente intensidad que su visión del mundo pudiese aportar a la percepción estética de la naturaleza, la explicación de la aflicción de Darwin debería resultar considerablemente llamativa.

En suma, la metáfora de "la orilla enmarañada" desarrollada en las últimas líneas del Origen, reflejo de una visión estrictamente y positivamente desencantada, está en equilibrio y colisión al mismo tiempo con una visión estrictamente aunque negativamente desencantada, representada por la metáfora del "daltonismo" en la Autobiografía. El desencantamiento weberiano no sería aplicable, aparentemente, al caso de Darwin, ya que este oscilaría entre la percepción de lo sublime perceptible gracias a actividades científicas y un irresoluble sentimiento de perceptibilidad estética parcial. Sin embargo, las interpretaciones que contemplan al naturalista como "intrínsecamente Romántico" a pesar de haberse separado del Romanticismo irracional ${ }^{35}$, no podrían extrapolarse del texto del Diario en donde sí se muestra (en ocasiones) una visión intrínsecamente "encantada" de la naturaleza. Por una parte, el Origen muestra una clara sensibilidad estética desencantada surgida a partir de un tipo de fuentes de inspiración tales como la consciencia de una actividad vital infinita en apariencia y minuciosa en esencia, no contemplada comúnmente por autores románticos. Por otra, el testimonio sobre la "atrofia" en una parte del cerebro y un supuesto indicio de "daltonismo"

33 Campbell, J. A., «Nature, Religion and Emotional Response: a Reconsideration of Darwin's Affective Decline», Victorian Studies №18 (2), Indiana University Press, 1974, pp. 161-162.

34 Levine, Darwin Loves You, op. cit., p. 146. Cursiva en el original.

35 Ídem. 
indicarían, en consecuencia, un sentimiento que en apariencia no se correspondería con lo expresado mediante la metáfora de "la orilla enmarañada".

Que la ciencia actual puede generar sentimientos de asombro y admiración del mundo gracias a que ofrece nuevas ópticas para aproximarse a él y comprenderlo mejor, no cabe duda. No obstante, la transición de Darwin a lo largo de su actividad científica que propongo, partiendo de una visión parcialmente "encantada" (en el Diario), pasando por un desencantamiento positivo (en el Origen) hasta alcanzar un desencantamiento negativo (en la Autobiografía), obligaría a asumir un proceso definitivamente unidireccional que hubiera partido de lo positivo y culminado en lo negativo. La disyuntiva (Entzauberung sí o no) en la interpretación de la percepción darwiniana de la naturaleza quedaría resuelta en la medida que se aceptara la imposibilidad de atribución de una única forma de percepción a Darwin, tanto la que enaltece la práctica científica para la percepción de lo sublime, como la que lo refuta.

Las últimas líneas del Origen confirman la capacidad de Darwin para percibir grandeza y belleza en la naturaleza ${ }^{36}$, compatible con la visión del mundo dada por la TE. Este hecho muestra, sin embargo, una contradicción con los pasajes de la Autobiografía sobre su "daltonismo" 37 perceptivo y su supuesta "atrofia cerebral"38. La sorprendente explicación de Darwin de estos dos fenómenos se podría deber a una incompleta o deficiente interpretación de sus estados mentales: dada la correlación entre la percepción, en la naturaleza, de la belleza, de lo sublime en ella, y del consiguiente sentimiento de lo sobrenatural, inferida a través de la rememoración del pasaje del Diario, que desvela la "convicción de que hay algo más en el ser humano que el mero aliento de su cuerpo"39 la pérdida del sentimiento religioso, en conjunción con la detección de una "atrofia" con respecto a las artes más finas, podría haber llevado a Darwin a deducir, tal vez erróneamente, una análoga pérdida de la capacidad de percepción estética en general, en lugar de considerarla como modificación de la percepción. No sólo el pasaje del "daltonismo", sino también el de la "atrofia cerebral" podría estar relacionado con la pérdida de percepción de lo sobrenatural en la naturaleza. Esto podría haber provocado en Darwin la sensación de haber perdido la sensibilidad estética. Sin embargo, esta hipótesis no excluye sostener que Darwin acabara teniendo, al final de su vida, una visión "desencantada” del mundo, en sentido weberiano.

\section{Referencias}

Barlow, N. (ed.) (2005), The Autobiography of Charles Darwin., 1809-1882, New York: W.W. Norton \& Company.

Campbell, J. A. (1974), «Nature, Religion and Emotional Response: a Reconsideration of Darwin's Affective Decline», Victorian Studies №18 (2), Indiana University Press.

Darwin, Ch. (1859), On the Origin of Species by Means of Natural Selection, or the Preservation of Favoured Races in the Struggle for Life, London: John Murray.

36 "There is grandeur in this view of life [...] endless forms most beautiful and most wonderful". Darwin, Origen, p. 490.

37 "I am like a man who has become colour-blind". Barlow (ed.), Autobiography, p. 76.

38 "why this should have caused the atrophy of that part of the brain alone". Ibíd., p. 113.

39 "I well remember my conviction that there is more in man than the mere breath of his body". Ibíd., p. 76. 
Darwin, Ch. (1839), Journal of Researches into the Natural History and Geology of the Various Countries Visited by H.M.S. Beagle round the World, under the Command of Capt. Fitz Roy, R.N., London: Henry Colburn.

Darwin, Ch. (1887), The Life and Letters of Charles Darwin, Including an Autobiographical Chapter, edited by Francis Darwin, London: John Murray.

Dawkins, R. (1998), Unweaving the Rainbow: Science, Delusion and the Appetite for Wonder, Boston: Houghton Mifflin Company.

Dennett, D. C. (1995), Darwin's Dangerous Idea: Evolution and the Meanings of Life, New York: Simon \& Schuster.

Holmes, R. (2009), The Age of Wonder: How the Romantic Generation Discovered the Beauty and Terror of Science, London: Harper Press.

Humboldt, A. von (1845-1862), Kosmos: Entwurf einer physischen Weltbeschreibung, Vol. I (publicado en cinco volúmenes), Stuttgart und Tübingen: Cotta.

Levine, G. (2008), Darwin Loves You: Natural Selection and the Re-enchantment of the World, Princeton (N.Y.): Princeton University Press.

Weber, M. (1992), La Ciencia como Profesión, traducción y edición de Joaquín Abellán, Madrid: Espasa Calpe. 